\title{
Recent progress on room temperature gas sensors for $\mathrm{NO}_{2}$ detection
}

\author{
Chao Zhang ${ }^{*}$, Xin Geng, Yifan Luo \\ College of Mechanical Engineering, Yangzhou University, Yangzhou 225127, China \\ *Corresponding Author: zhangc@yzu.edu.cn
}

\begin{abstract}
Air pollution is nowadays a big issue regarding the survival of mankind. The development of high-performance, reliable and low-cost $\mathrm{NO}_{2}$ sensors are urgently needed. Recently, semiconductor gas sensors that do not need any heating element for high-temperature operation have received extensive attention. These sensors present many advantages: low power consumption, low manufacturing cost, as well as moderate stability and safety. Typically, wide bandgap semiconductors have weak, very slow or no response to $\mathrm{NO}_{2}$ gases at room temperature. Therefore, some enhancing methods like light illumination, organic sensitization, formation of heterojunctions, preparation of composites with 2D materials and introduction of oxygen vacancies in high concentrations have been used to improve the sensing performance according to the published works. These methods and research progress are presented and discussed in this paper.
\end{abstract}

Keywords: Gas sensors, Room temperature, Nitrogen dioxide

\section{Introduction}

Nitrogen dioxide $\left(\mathrm{NO}_{2}\right)$ is a toxic gas produced from the combustions of coal, gas, oil or wood for industrial and domestic use. Internal combustion engines used in vehicles can generate $\mathrm{NO}_{2}$ as well. $\mathrm{NO}_{2}$ can harm human health when its concentration is beyond $200 \mu \mathrm{g} / \mathrm{m}^{3}$. It can also cause severe environmental problems such as acid rain and fog $^{(1-2)}$. To tackle this problem, the first thing to do is to monitor its concentration. Therefore, it is essential to develop $\mathrm{NO}_{2}$ monitoring technique which is highly sensitive, low cost and easy maintained.

Metal oxide semiconductors (MO) are the most widely-used $\mathrm{NO}_{2}$ sensing materials. Compared with other gas analyzers, MO gas sensors have advantages, including low cost, small size and easy maintained ${ }^{(3)}$. However, this kind of gas sensors has some shortcomings, such as poor selectivity and high power consumption.

Operating temperature is a critical factor which affects the sensing performance of MO gas sensors. It determines the reaction type and rate of gases at the surface of sensors and shows an impact on sensitivity, response time and energy consumption of the sensors. Moreover, working at high temperature involves a complexity in the design of the sensors to achieve long-term stability (the substrate must withstand the high temperature for a long period). Reducing the operating temperature not only results in low power consumption but simplifies the construction of the sensors, and also enhances the stability of the sensing materials. The reduction of operating temperature can also improve the safety and usability under complex gas condition (flammable gases or gases which can dissociate at high temperature). So, it has become a significant research objective to lower the operating temperature of semiconductor gas sensors to room temperature.

Our group has done some works in the area of room temperature $\mathrm{NO}_{2}$ sensors, such as light illumination, organic sensitization, forming $\mathrm{p}-\mathrm{n}$ heterojunctions and introducing high concentration oxygen vacancies. These works will be introduced below. Also, an outlook of the key points in the study of room temperature gas sensors will be presented.

\section{UV / Visible light illumination}

Light illumination is an effective method to reduce the operating temperature of $\mathrm{MO} \mathrm{NO} \mathrm{NO}_{2}$ sensors. The energy which is used to excite the electron-hole pairs will be supplied by the photons instead of heat. According to the formula $\mathrm{E}=\mathrm{hc} / \lambda$, it is possible to calculate the wavelength which is suitable to excite the electrons to higher states ${ }^{(4)}$. The bandgap of most MO used as sensing materials is 
higher than $3 \mathrm{eV}$, which means that they can only be excited by UV light. However, UV light illumination can bring some new problems. UV light needs a customized light source, which makes it difficult to be used under special conditions. Also, UV light can result in the decomposition or combination reaction of target gases. The relatively high photon energy of UV light can also cause damage to some of the sensors. All these shortcomings limit the application of UV light, so it is necessary to develop room temperature gas sensors which can be activated by visible light.

The wavelength range of visible light is 390-780 nm, implying that only those semiconductors with a bandgap of 2.55-3.18 eV can absorb visible light. So it is necessary to adjust the bandgap structure of $\mathrm{MO} \mathrm{NO}_{2}$ sensors. Our group firstly fabricated $\mathrm{WO}_{3}$ films on the substrates of sensors (aluminum oxide with Au interdigital electrode) by screen printing and tested its response to 160 and $320 \mathrm{ppb} \mathrm{NO}_{2}$ under the irradiation of visible light with different wavelengths ${ }^{(5-6)}$. They successfully proved that visible light illumination could effectively improve the response ability of $\mathrm{WO}_{3}$ films to $\mathrm{NO}_{2}$ gas at room temperature. They also found that the wavelength and the intensity of visible light have a significant influence on the sensing ability of $\mathrm{WO}_{3}$. When exposed to blue light, the $\mathrm{WO}_{3}$ sensor has the best response and recovery time, and the sensing ability is similar to the result under $200^{\circ} \mathrm{C}$, as is shown in Fig. 1 .

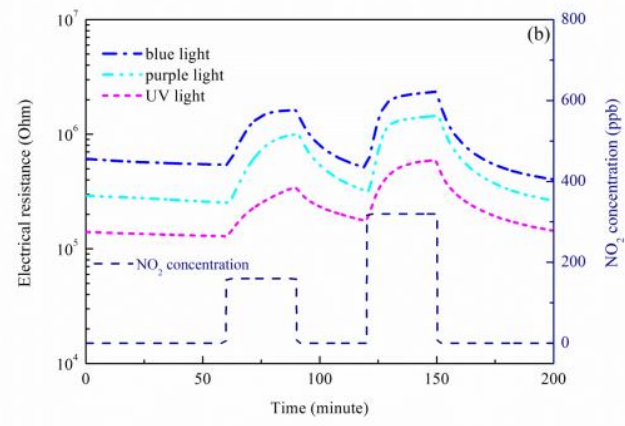

Fig. 1. Electrical resistance responses of $\mathrm{WO}_{3}$ films submitted to 160 and $320 \mathrm{ppb} \mathrm{NO}$ illuminated by blue, purple and UV lights ${ }^{(5)}$.

\section{Organic sensitization}

Since the visible light cannot be absorbed by wide bandgap semiconductors such as $\mathrm{ZnO}$ and $\mathrm{SnO}_{2}$, we tried to use organic dyes to reduce the bandgap and expand the absorption range to visible light. N719 is a commonly used $\mathrm{Ru}$ complex in DSSC, so we tried to immerse $\mathrm{ZnO}$ films into N719 solution and successfully expand its light absorption range to visible light ${ }^{(7)}$. The base resistance of amorphous $\mathrm{ZnO}$ decreased significantly under the irradiation of visible light. The sensors showed a significant response to $1.25-10 \mathrm{ppm} \mathrm{NO}_{2}$.



Fig. 2. UV-visible diffuse reflectance spectra of (a) $\mathrm{Al}_{2} \mathrm{O}_{3}$ substrate (b) a-ZnO film and (c) N719-dye-sensitized a-ZnO film at room temperature ${ }^{(7)}$.

\section{4. p-n heterojunctions}

Using p-type semiconductors with narrow bandgap to expand the light absorption range is another effective method. Here, the p-type materials are used to absorb visible light while the n-type semiconductors are used as gas sensing material. When the photon energy is higher than the width of the bandgap, electrons in the valence band will be excited and transferred into the conductance band. The surface conductivity will change significantly. Because of that, the reaction at the surface will be accelerated and then improve the gas sensing ability at room temperature. We deposited $\mathrm{CdS}-\mathrm{ZnO}$ coatings on $\mathrm{Al}_{2} \mathrm{O}_{3}$ substrates by liquid plasma spray method ${ }^{(8)}$. When the sensors were exposed to visible lights with a different wavelength, the result indicated that the highest response was measured under the illumination of green light, the response was 31.9 to $1 \mathrm{ppm} \mathrm{NO}_{2}$ while the response time of $16.7 \mathrm{~min}$.



Fig. 3. UV-visible diffuse reflectance spectra of pure $\mathrm{ZnO}$ and $\mathrm{CdS}-\mathrm{ZnO}{ }^{(8)}$. 


\section{Composite with 2D materials}

2D materials such as graphene have some special properties. Pure graphene does not have a bandgap. Its electrical properties are similar to conductive materials, which means that the pure graphene cannot fit with semiconductors well. So, some methods such as cutting the graphene into several nanometers wide nanostripes or providing some oxygen-containing functional groups on it to change the electronic structure of graphene and make it becomes a semiconductor. GO showed a p-type property and fast electron transfer rate, which is beneficial to the gas sensing performance. We prepared $\mathrm{GO}-\mathrm{WO}_{3}$ sensing layer by dipping $\mathrm{WO}_{3}$ coatings in the $\mathrm{GO}$ suspension ${ }^{(9)}$. The sensor showed an extremely high response to $0.9 \mathrm{ppm} \mathrm{NO}_{2}$ under the irradiation of green light. The sensing performance is showed clearly in Fig. 4.

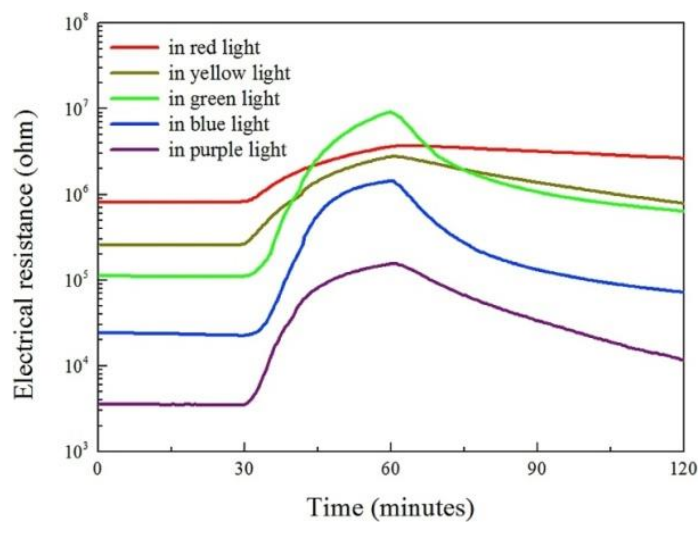

Fig. 4. The electrical resistance responses of the $\mathrm{WO}_{3}-\mathrm{GO}$ sensors to $0.9 \mathrm{ppm} \mathrm{NO}_{2}$ gas under light illumination at different wavelengths at room temperature ${ }^{(9)}$.

\section{Oxygen vacancy modification}

Introducing defects, especially oxygen vacancies into semiconductors is a novel method to enhance the semiconductors' photon absorption and gas adsorption performance. Oxygen vacancies not only can provide the adsorption sites for oxidizing gases like $\mathrm{NO}_{2}$, but also can change the structure of semiconductors' bandgap, which was confirmed by DFT calculation ${ }^{(10)}$. The oxygen vacancies existed in the MO sensor play the role of deep donors. The electrons in the deep donors are easier to be excited to the conduction band. When exposed to visible light or heated up, the electrons in the deep donors will be excited into the conductance band first and change the electrical resistance. The surface activity of semiconductors increases with the increase of the concentration of oxygen vacancies. Therefore, introducing high concentration oxygen vacancies into MO sensors can significantly increase its gas sensing ability. This method does not need additional photosensitizer and will not cause the decrease of sensors' sensitization or add the difficulties in the production process. Our group fabricated $\mathrm{ZnO}_{1-\mathrm{x}}$ coatings with high concentration oxygen vacancies by liquid plasma spray method. It showed a high response to $0.9 \mathrm{ppm} \mathrm{NO}_{2}$.

Also, we have tried to prepare composite sensing layers with high concentration oxygen vacancies. $\mathrm{ZnO}-\mathrm{CdS}$, $\mathrm{Cu}_{\mathrm{x}} \mathrm{O}_{1-\mathrm{y}} @ \mathrm{ZnO}_{1-\alpha}$ and $\mathrm{SnO}_{1-\alpha} @ \mathrm{ZnO}_{1-\beta} @ \mathrm{SnO}_{2-\gamma}$ are prepared by liquid plasma spray method ${ }^{(11-13)}$. The $\mathrm{ZnO}-\mathrm{CdS}$ coatings are treated by $\mathrm{H}_{2} \mathrm{O}_{2}$ after the spray process. All these sensors showed a much higher response to $\mathrm{NO}_{2}$, as is shown in Fig. 5.
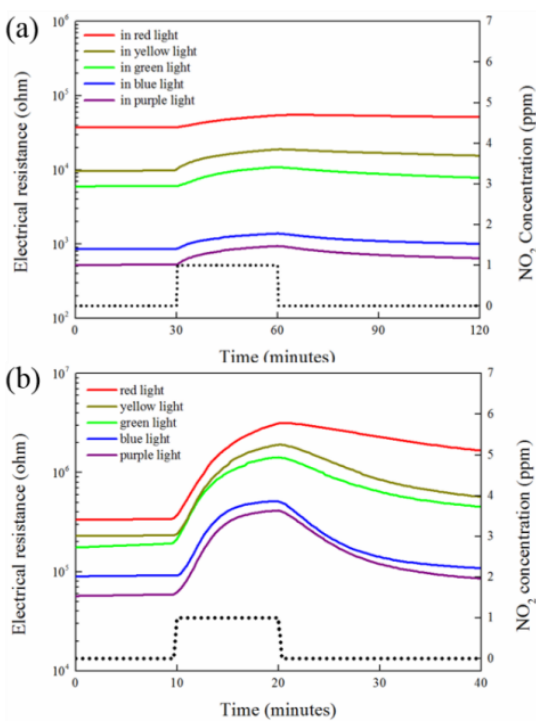

Fig. 5. $\mathrm{NO}_{2}$ response of $\mathrm{ZnO}$ coatings (a) before and (b) after $\mathrm{H}_{2} \mathrm{O}_{2}$ treatment. (11)

\section{Summary and Outlook}

With the development of the fabricating and enhancing technologies in semiconductors and gas sensors, MO gas sensors are now spreading in the direction of low operation temperature, integration and low energy consumption. Reducing the operating temperature to room temperature is of great importance in the field of the stability, structure and power consumption of the sensors.

However, its selectivity and stability still need to be improved. In addition, room temperature semiconductor $\mathrm{NO}_{2}$ sensors still need to face problems like long response/recovery time, low repeatability, etc. These problems should be solved by improving the fabrication 
process of sensing materials and innovation of new materials system, such as the synthesize of new semiconductors with special morphologies which can provide more adsorption sites. Also, the effect of humidity on MO gas sensors at room temperature is a huge challenge. As far as I concerned, this problem may be solved by adding a thin superhydrophobic film which can let the gas molecules get through as well. Until now, many useful works like fabricating $\mathrm{p}-\mathrm{n}$ heterojunctions, organic/inorganic activation, developing new nanostructures and increasing the concentration of oxygen vacancies have been launched. However, there are still many works to be done in these areas. These works are meaningful for obtaining fast response and recovery rate, as well as improving the stability and selectivity.

\section{References}

(1) J. Zhang, J. Wu, X. Wang, D. Zeng, C. Xie. Enhancing room-temperature $\mathrm{NO}_{2}$ sensing properties via forming heterojunction for NiO-rGO composited with $\mathrm{SnO}_{2}$ nanoplates. Sens. Actuat. B: Chem. 243 (2017) 1010-1019.

(2) C. Zhang, M. Debliquy, A. Boudiba, H. Liao, C. Coddet. Sensing properties of atmospheric plasma-sprayed $\mathrm{WO}_{3}$ coating for sub-ppm $\mathrm{NO}_{2}$ detection. Sens. Actuat. B: Chem. 144 (2010) 280-288.

(3) G. Eranna, B. C. Joshi, D. P. Runthala, R. P. Gupta. Oxide Materials for Development of Integrated Gas Sensors-A Comprehensive Review. Critical Reviews in Solid State and Materials Sciences. 29 (2004) 111-188.

(4) Y. Luo, C. Zhang, B. Zheng, X. Geng, M. Debliquy. Hydrogen sensors based on noble metal doped metal-oxide semiconductor: A review. Int. J. Hydrogen Energy. 42 (2017) 20386-20397.

(5) C. Zhang, A. Boudiba, P. Marco, R. Snyders, M. Olivier, M. Debliquy. Room temperature responses of visible-light illuminated $\mathrm{WO}_{3}$ sensors to $\mathrm{NO}_{2}$ in sub-ppm range. Sens. Actuat. B: Chem. 181 (2013) 395-401.

(6) C. Zhang, A. Boudiba, C. Bittencourt, R. Snyders, M.
Olivier, M. Debliquy. Visible Light Activated Tungsten Oxide Sensors for NO2 Detection at Room Temperature. Procedia Engineering. 47 (2012) 116-119.

(7) C. Zhang, J. Wang, M. Olivier, M. Debliquy. Room temperature nitrogen dioxide sensors based on N719-dye sensitized amorphous zinc oxide sensors performed under visible-light illumination. Sens. Actuat. B: Chem. 209 (2015) 69-77.

(8) X. Geng, C. Zhang, M. Debliquy. Cadmium sulfide activated zinc oxide coatings deposited by liquid plasma spray for room temperature nitrogen dioxide detection under visible light illumination. Ceramics International, 42 (2016) 4845-4852.

(9) X. Geng, J. You, J. Wang, C. Zhang. Visible light assisted nitrogen dioxide sensing using tungsten oxide-Graphene oxide nanocomposite sensors. Materials Chemistry and Physics. 191 (2017) 114-120.

(10) C. Zhang, X. Geng, J. Li, Y. Luo, P. Lu. Role of oxygen vacancy in tuning of optical, electrical and $\mathrm{NO}_{2}$ sensing properties of $\mathrm{ZnO}_{1-\mathrm{x}}$ coatings at room temperature. Sens. Actuat. B: Chem. 248 (2017) 886-893.

(11) X. Geng, J. You, C. Zhang. Microstructure and sensing properties of $\mathrm{CdS}-\mathrm{ZnO}_{1-\mathrm{x}}$ coatings deposited by liquid plasma spray and treated with hydrogen peroxide solution for nitrogen dioxide detection at room temperature. Journal of Alloys and Compounds. 687 (2016) 286-293.

(12) X. Geng, C. Zhang, Y. Luo, M. Debliquy. Preparation and characterization of $\mathrm{CuxO}_{1-\mathrm{y}} @ \mathrm{ZnO}_{1-\alpha}$ nanocomposites for enhanced room-temperature $\mathrm{NO}_{2}$ sensing applications. Applied Surface Science. 401 (2017) 248-255.

(13) X. Geng, C. Zhang, Y. Luo, H. Liao, M. Debliquy. Light assisted room-temperature $\mathrm{NO}_{2}$ sensors with enhanced performance based on black $\mathrm{SnO}_{1-\alpha} @ \mathrm{ZnO}_{1-\beta} @ \mathrm{SnO}_{2-\gamma}$ nanocomposite coatings deposited by solution precursor plasma spray. Ceram. Int. 43 (2017) 5990-5998. 